\title{
A self-assembled microlensing rotational probe
}

\author{
James P. Brody and Stephen R. Quake ${ }^{\text {a) }}$ \\ Department of Applied Physics, California Institute of Technology, Pasadena, California 91125
}

(Received 14 September 1998; accepted for publication 30 October 1998)

\begin{abstract}
A technique to measure microscopic rotational motion is presented. When a small fluorescent polystyrene microsphere is attached to a larger polystyrene microsphere, the larger sphere acts as a lens for the smaller microsphere and provides an optical signal that is a strong function of the azimuthal angle. We demonstrate the technique by measuring the rotational diffusion constant of the microsphere in solutions of varying viscosity and discuss the feasibility of using this probe to measure rotational motion of biological systems. (C) 1999 American Institute of Physics.
\end{abstract}

[S0003-6951(99)03401-4]

The commercial development of precision microspheres with well controlled sizes and various protein coatings has enabled a number of new devices and experimental techniques, from being the component parts in the fabrication of photonic crystals ${ }^{1}$ to their use as "handles" for optical tweezers in the study of single protein ${ }^{2,3}$ and deoxyribose nucleic acid (DNA) molecules. ${ }^{4}$ Here we describe the use of microspheres with streptavidin-biotin coating to self-assemble a microscopic lensing system that can in turn be used to measure rotation.

Previous experiments in chemical physics have measured bulk molecular rotational diffusion with high time resolution using fluorescence anisotropy decay ${ }^{5}$ and fluorescence correlation spectroscopy; ${ }^{6}$ however, interpretation of the experimental data is a delicate issue. ${ }^{5}$ Biological experiments to measure rotational motions used a "tethered cell" assay in which a single flagellum is attached to a surface and the cell rotates ${ }^{7-9}$ or a "rotating filament" assay by attaching long actin $^{10}$ filaments to an ATPase molecule or microtubule ${ }^{11}$ filaments to a dynein molecule. Both these assays rely on imaging the probe to determine orientation, limiting the time resolution to video rates on the order of $10 \mathrm{~Hz}$.

Although it is inferred from the swimming speed of microorganisms that bacterial flagella can turn at a top rotational speed of $100000 \mathrm{rpm},{ }^{12}$ measurement techniques cannot keep up with those rates. Measurements are typically made by forcing the flagella to turn a large moment of inertia which slows the rotation rate to the region where it is accessible to video analysis. Similarly, in studies of $F_{1}$-ATPase, the rotation rate is slowed to an observable level by increasing the hydrodynamic drag that it must oppose. However, the rotation is likely to be fundamentally at a constant angle per ATP hydrolized, as shown by Refs. 13 and 14, and slowing the rotation by increasing drag results in some elastic storage of energy. Methods are needed that have the potential to observe high-frequency rotations and have less hydrodynamic drag.

This letter presents a method to measure high-frequency rotational motion. An asymmetric fluorescent probe is made by attaching a small fluorescently labeled polymer microsphere to a larger polymer microsphere. The larger sphere

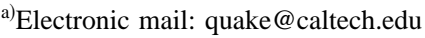

acts as a lens, substantially enhancing the collection efficiency of the optical system. The experiment is outlined in the inset of Fig. 1.

We can show that the system increases the amount of collected light with a geometric optics argument. The angles of ray tracing are outlined in Fig. 1; we would like to calculate the exit angle $\phi^{\prime}-\theta^{\prime \prime}$ as a function of the incident angle $\theta$. The small fluorescent microsphere is approximated as a point particle a distance $\delta$ from the lensing microsphere. Using geometry, note that

$$
\phi^{\prime}=\pi-\left(\pi-2 \theta^{\prime}+\phi\right)=2 \theta^{\prime}-\phi .
$$

Applying Snell's law at the top interface of the lensing microsphere we find that

$$
\theta^{\prime \prime}=\sin ^{-1}\left(\frac{n_{2}}{n_{1}} \sin \theta^{\prime}\right)
$$

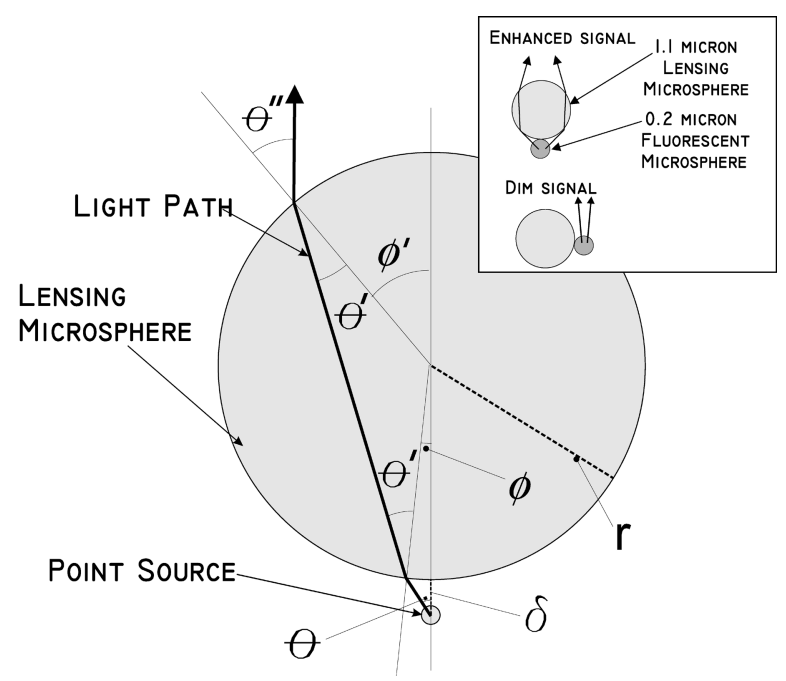

FIG. 1. The optical ray tracing of the two microspheres. This diagram defines the angles $\theta, \theta^{\prime}, \theta^{\prime \prime}, \phi, \phi^{\prime}$ and the distances $r$ and $\delta$. The ray originally starts at an angle $\theta$ to the vertical and after passing through the lensing microsphere continues on at an angle $\phi^{\prime}-\theta^{\prime \prime}$. The index of refraction of the water is $n_{1}=1.3$ and for the polystyrene microsphere is $n_{2}=1.59$. (Inset) The fluorescence collected from an objective with finite NA is enhanced when the microsphere pair is aligned with the optical collection axis. 
where $n_{2}$ is the index of refraction of the lensing microsphere and $n_{1}$ is the index of refraction of the surrounding medium (typically water). Applying Snell's law at the bottom interface we obtain

$$
\theta^{\prime}=\sin ^{-1}\left(\frac{n_{1}}{n_{2}} \sin (\theta+\phi)\right)
$$

Then, by direct substitution of Eq. (3) into Eq. (2), we find that

$$
\theta^{\prime \prime}=\phi+\theta .
$$

Using the law of sines, we can write

$$
\frac{\sin (\pi-\theta-\phi)}{r+\delta}=\frac{\sin \theta}{r},
$$

and then explicitly find the angle $\phi$ as a function of $r, \theta$, and $\delta$ :

$$
\phi(r, \theta, \delta)=\sin ^{-1}\left(\frac{r+\delta}{r} \sin \theta\right)-\theta .
$$

Finally, we can write the exit angle $\phi^{\prime}-\theta^{\prime \prime}$ in terms of the original angle $\theta$, the radii of the two spheres $r, \delta$, and the indices of refraction $n_{1}$ and $n_{2}$ :

$\phi^{\prime}-\theta^{\prime \prime}=2 \sin ^{-1}\left(\frac{n_{1}}{n_{2}} \sin [\theta+\phi(r, \theta, \delta)]\right)-\theta-2 \phi(r, \theta, \delta)$.

For $\delta \ll r$, we note that $\phi \ll \theta$. The exit angle is then given by

$$
\phi^{\prime}-\theta^{\prime \prime}=2 \sin ^{-1}\left(\frac{n_{1}}{n_{2}} \sin \theta\right)-\theta .
$$

Typical realizable values of $n_{1}$ and $n_{2}$ are for water $n_{1}$ $=1.3$, and polystyrene $n_{2}=1.59$. For small $\theta$ Eq. (8) reduces to $\left[2\left(n_{1} / n_{2}\right)-1\right] \theta$. This gives an exit angle of $0.64 \theta$ for a ray entering at an angle $\theta$. Since the exit angle is always less than the original angle, we conclude that the lensing microsphere focuses rays from the fluorescent microsphere and enhances the optical signal.

The enhancement in the observed optical signal also depends on the numerical aperture (NA) of the objective. The NA is defined as NA $=n \sin \theta_{0}$, where $\theta_{0}$ is the collection angle. For our objective $(20 \times, 0.4 \mathrm{NA})$ in air $\theta_{0}=23.6^{\circ}$. Equation (8) shows that the focusing microsphere increases the angle of collection to $43.5^{\circ}$. This corresponds to an effective NA of 0.69. The epifluorescent intensity is proportional to $\mathrm{NA}^{4}$, so we expect an intensity enhancement of $(0.69 / 0.4)^{4} \approx 9$ times; this is of the order of what we observed. Objectives with high NA collect almost all of the emitted light, and thus we would not expect to see any fluorescent enhancement from a high NA objective. Observations made with a $40 \times, 1.30 \mathrm{NA}$ oil immersion objective indicate no noticeable intensity enhancement.

To construct this system we used the biotin/streptavidin binding system. Biotin is known to bind streptavidin with a very high affinity. ${ }^{15}$ We obtained streptavidin coated microspheres from Interfacial Dynamics (polystyrene at a volume concentration of $2.6 \%, 1.1 \mu \mathrm{m}$ diameter) and biotin coated yellow-green fluorescent microspheres (polystyrene $1 \%$ by volume, $0.2 \mu \mathrm{m}$ diameter, excitation maximum at $505 \mathrm{~nm}$, emission maximum at $515 \mathrm{~nm}$ ) from Molecular Probes. The smaller microspheres were diluted by a factor of 1000 in distilled water. A volume of $10 \mu \mathrm{l}$ of this solution was added to $10 \mu \mathrm{l}$ of the large microspheres and allowed to incubate at room temperature for approximately $5 \mathrm{~min}$. The solution was then diluted by a factor of $10^{4}$ in either distilled water or a glycerol/water mixture to give a final concentration of approximately 1 microsphere pair per nanoliter at three different viscosities $(1,4$, and $13 \mathrm{cP})$.

The solution was placed on a microscope slide with a coverslip and the edges were sealed with a clear nail polish to prevent any evaporation or fluid flow. These specimens were examined on a Nikon fluorescent microscope with a 20 $\times, 0.4 \mathrm{NA}$ objective. The lensing microsphere undergoes constant rotational diffusion, and when the attached fluorescent microsphere is aligned with the optical collection axis the observed intensity is substantially larger than in other positions.

Images of these intensity fluctuations were observed using a Phillips FTM-800 charge coupled device (CCD) camera and recorded on video tape. The sequence of video images was analyzed by digitizing them with a $\mathrm{PC}$ at a $30 \mathrm{~Hz}$ frame rate. The microspheres' fluorescent intensities were measured in each frame, and their translation diffusion was slow enough that their positions could be followed from frame to frame. Data with better time resolution could be obtained by using a photodiode or photomultiplier tube to observe single microspheres, but the video camera is sufficiently fast for observing rotational diffusion and offered the advantage of being able to observe many microsphere pairs in parallel.

From the intensity fluctuations we can compute the probability $P(t)$ that the intensity is above a threshold at time $t$, given that the intensity was above this threshold at time $t$ $=0 . P(t)$ can in turn be calculated from first principles. The rotational motion of the lensing microsphere can be decomposed into orthogonal azimuthal and polar directions. Since only the azimuthal angle contributes to the intensity enhancement, it suffices to consider a one-dimensional problem. Let $\alpha$ denote the azimuthal angle over which the signal is enhanced, which then determines the initial conditions. The solution for the one-dimensional diffusion equation with initial condition $|\theta| \leqslant \alpha$ and infinite boundary conditions is ${ }^{16}$

$$
p_{\text {inf }}(\theta, t)=\frac{1}{2 \alpha}\left[\operatorname{erf}\left(\frac{\alpha / 2-\theta}{2 \sqrt{D_{r} t}}\right)+\operatorname{erf}\left(\frac{\alpha / 2+\theta}{2 \sqrt{D_{r} t}}\right)\right],
$$

where $D_{r}$ is the rotational diffusion constant of a sphere

$$
D_{r}=\frac{k_{B} T}{8 \pi \eta a^{3}},
$$

$\eta$ is the viscosity and $a$ is the sphere's radius. ${ }^{17}$ Equation (9) can be made periodic in $\theta$ by summing over all multiples of $2 \pi$. The probability density function for the distribution in angles is then

$$
p(\theta, t)=\sum_{n=-\infty}^{n=\infty} p_{\text {inf }}(\theta+2 n \pi, t)
$$




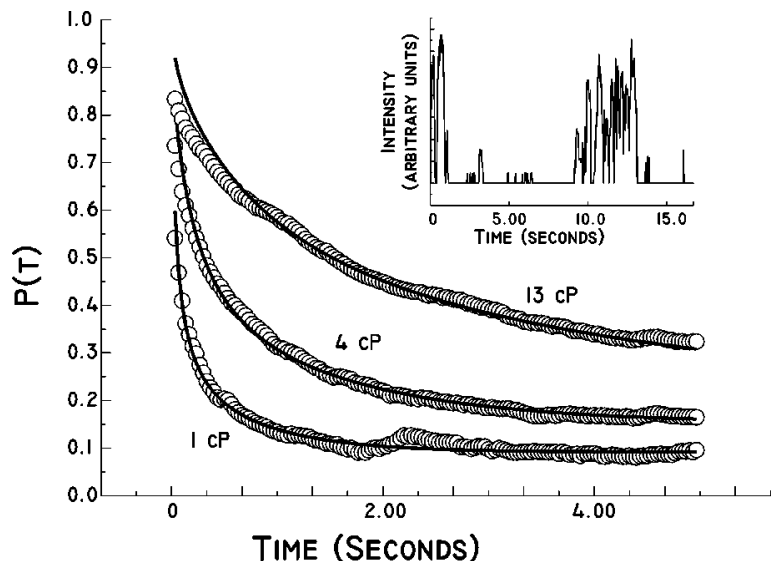

FIG. 2. The probability correlation function of the fluorescent intensity signal measured in three different glycerol-water solutions of varying viscosities. These measurements are the average over approximately 30 microspheres measured over approximately $1 \mathrm{~min}$. The errors in the measurements are on the order of the diameter of the circles marking the data points. The solid lines represent fits to the data with the following parameters: $\quad D_{1}=1.30( \pm 0.03) \mathrm{s}^{-1}, \quad \alpha_{1}=32.7( \pm 0.2)^{\circ}, \quad D_{4}=0.70$ $( \pm 0.02) \mathrm{s}^{-1}, \quad \alpha_{4}=55.0( \pm 0.5)^{\circ}, \quad D_{13}=0.100( \pm 0.003) \mathrm{s}^{-1}, \quad \alpha_{13}=46.5$ $( \pm 1.1)^{\circ}$. The inset shows typical data from a single microsphere $(\eta \approx 4 \mathrm{cP})$ over $18 \mathrm{~s}$.

$$
P(t)=\int_{-\alpha / 2}^{\alpha / 2} p(\theta, t) d \theta .
$$

In practice this was approximated numerically using just the largest terms of the series $(|n| \leqslant 3)$. The $n=0$ term is computed using a Chebyshev approximation to the error function and integrating it using a trapezoid algorithm. ${ }^{18}$ The higher order terms were computed using the approximation that the initial condition was $p(\theta, 0)=\delta(\theta)$ (the solution is a Gaussian function) and the integration was carried out as the value of the function times the width.

The data can therefore be fit to a two parameter function $P(t)$ that is characterized by the angle $\alpha$ and the rotational diffusion coefficient $D_{r}$. The angle $\alpha$ is determined by the optics of the system and the threshold applied to the data. The rotational diffusion coefficient $D_{r}$ is given by Eq. (10). The measured diffusion coefficients are in agreement with the predicted values (Fig. 2).

We have outlined a mechanism by which a microlensing effect is used to measure rotations of individual micro- spheres. The microlensing can be explained with geometric optics; a complete theory will need to take into account near field optical effects. The microlensing was observed experimentally and used to measure the rotational diffusion constant of a sphere. This mechanism may have applications in studies of biomolecular rotations and fluid dynamics, especially in situations with a high rotational rate. For a rotation at a constant rate, the load can be adjusted by changing both the viscosity of the fluid and size of the larger microsphere. Precision microspheres are commercially available with diameters of less than $1 \mu \mathrm{m}$ to greater than $100 \mu \mathrm{m}$ giving a range of loads that differ by $10^{6}$.

This work was supported by the Whitaker Foundation and by NSF CAREER Grant No. PHY-9722417.

${ }^{1}$ H. Miguez, C. Lopez, F. Meseguer, A. Blanco, L. Vazquez, R. Mayoral, M. Ocana, V. Fornes, and A. Mifsud, Appl. Phys. Lett. 71, 1148 (1997).

${ }^{2}$ K. Svoboda, C. F. Schmidt, B. J. Schnapp, and S. M. Block, Nature (London) 365, 721 (1993).

${ }^{3}$ J. T. Finer, R. M. Simmons, and J. A. Spudich, Nature (London) 368, 113 (1994).

${ }^{4}$ T. T. Perkins, S. R. Quake, D. E. Smith, and S. Chu, Science 264, 822 (1994).

${ }^{5}$ D. M. Jameson and T. L. Hazlett, in Biophysical and Biochemical Aspects of Fluorescence Spectroscopy, edited by T. G. Dewey (Plenum, New York, 1991), p. 105.

${ }^{6}$ E. Elison, Annu. Rev. Phys. Chem. 36, 379 (1985).

${ }^{7}$ R. M. Berry and H. C. Berg, Proc. Natl. Acad. Sci. USA 94, 14433 (1997).

${ }^{8}$ H. Berg, Nature (London) 249, 77 (1974).

${ }^{9}$ M. Silverman and M. Simon, Nature (London) 249, 73 (1974).

${ }^{10}$ C. Shingyoji, H. Higuchi, M. Yoshimura, E. Katayama, and T. Yanagida, Nature (London) 393, 711 (1998).

${ }^{11}$ K. Kinoshita, R. Yasuda, H. Noji, S. Ishiwata, and M. Yoshida, Cell 93, 21 (1998).

${ }^{12}$ D. J. DeRosier, Cell 93, 17 (1998).

${ }^{13}$ H. Noji, R. Yasuda, M. Yoshida, and K. Kinosita, Nature (London) 386, 299 (1997).

${ }^{14}$ R. Yasuda, H. Noji, K. Kinosita, and M. Yoshida, Cell 93, 1117 (1998).

${ }^{15}$ Avidin-biotin Technology, edited by M. Wilchek and E. A. Bayer (Academic, San Diego, CA, 1990).

${ }^{16}$ J. Crank, Mathematics of Diffusion (Oxford University Press, London, 1970).

${ }^{17}$ T. G. M. van de Ven, Colloidal Hydrodynamics (Academic, San Diego, CA, 1989)

${ }^{18}$ W. H. Press, S. A. Teukolsky, W. T. Vetterling, and B. P. Flannery, Numerical Recipes in C:The Art of Scientific Computing (Cambridge University Press, New York, 1993). 